\title{
Evolution of dispersal distance
}

\author{
Rick Durrett* and Daniel Remenik ${ }^{\dagger \dagger}$
}

May 18, 2011

\begin{abstract}
The problem of how often to disperse in a randomly fluctuating environment has long been investigated, primarily using patch models with uniform dispersal. Here, we consider the problem of choice of seed size for plants in a stable environment when there is a trade off between survivability and dispersal range. Ezoe (1998) and Levin and Muller-Landau (2000) approached this problem using models that were essentially deterministic, and used calculus to find optimal dispersal parameters. Here we follow Hiebeler (2004) and use a stochastic spatial model to study the competition of different dispersal strategies. Most work on such systems is done by simulation or nonrigorous methods such as pair approximation. Here, we use machinery developed by Cox, Durrett, and Perkins (2011) to rigorously and explicitly compute evolutionarily stable strategies.
\end{abstract}

\section{Introduction}

"To disperse or not to disperse?," that was the first question. There was much work on this topic in the 1970s and early 1980s, see e.g., Hamilton and May (1977), who proved the surprising result that no matter how costly dispersal is, the optimal strategy involves some dispersal. Levin, Cohen, and Hastings (1984) began the development of analytical results necessary to understand the results of numerical experiments. They considered an environment made up of $L$ distinct sites. Dispersing seeds are lost with probability $1-\alpha$ with the survivors spread uniformly over patches, while nondispersing seeds have a mortality $1-v$. As is now common in the analysis of

*Dept. of Math, Duke U., Box 90320, Durham NC 27708-0320, Phone: (919) 660-6970, Fax: (919) 660-2821, Email: rtd@math.duke.edu

${ }^{\dagger}$ Dept. of Math, U. of Toronto, 40 St. George St., Toronto, Ontario CANADA M5S 2E4 and Depto. de Ing. Matem., U de Chile, Av. Blanco Encalada 2120 Santiago, Chile

$\ddagger$ This work was done while the authors were at Cornell University and patially supported by NSF grant DMS 0704996 from the probability program. 
these questions they sought an evolutionarily stable strategy (ESS), i.e., one that once established cannot be invaded.

Cohen and Levin (1991) followed up on this work by considering environments that were temporally correlated and spatially heterogeneous. They found and computed ESS's in the case of nonnegative correlations of environmental quality, but found in some cases with negative correlation that there was no ESS. Ludwig and Levin (1991) solved this problem by showing that there were evolutionary stable combinations of dispersal types that could not be invaded.

The last two paragraphs, which highlight some of the early work of our honoree, do not begin to do justice to the vast literature on this topic. Our treatment of this topic is brief because we will be concerned with the problem of optimal dispersal range. Ezoe (1988) was the first to tackle this question. He considered the optimal choice of seed size. Larger seeds are beneficial in the competition for safe sites, but are likely to be dispersed a shorter distance and to suffer competition with siblings. He supposed that dispersal followed a two dimensional normal distribution where the variance and the average number of seeds dispersed are decreasing functions of the seed size.

Levin and Muller-Landau (2000) followed up on this work and assumed that the each genotype produces seeds of a single weight $w$. They let $n(w)$ be the number of seeds produced, let $c(w)$ be their relative competitive advantage, and let $\delta(w, x)$ be the probability a seed is dispersed $x$ from its source. They noticed that the "potency" $P=c(w) n(w)$ and the function

$$
F=P^{2}(w)\left[1-\sum_{x} \delta(w, x)^{2}\right]
$$

were important. In particular, maxima of $F$ were convergence stable strategies, that is, ESS towards which selection will drive populations. See Levin, Muller-Landau, Nathan, and Chave (2003) for a survey of this and subsequent developments.

Ezoe (1988) and Levin and Muller-Landau (2000) treat seeds as continuous quantities that are spread according to a density function. Hiebeler (2004) investigated a model with discrete patches at the points of the two-dimensional square lattice which can be in one of three states: $0=$ empty suitable habitat, $1=$ empty unsuitable habitat, and $2=$ occupied suitable habitat. He studied the competition of near (nearest neighbor) and far (global over the finite set being simulated) dispersal strategies using simulation and the pair-approximation.

\section{$2 \quad$ Model and Results}

In this paper we also consider a spatial model on the two dimensional lattice. However rather than considering a multi-type contact process as Hiebeler did, we will follow Neuhauser and Pacala (1999) and study the much simpler system in which each site 
is always occupied by one of two competing species. We use 0 and 1 to indicate sites occupied by two different types. This use of 0 and 1 is unfortunate because it may make the reader think of vacant and occupied but it is notation used by both Cox, Durrett, Perkins (2011) and Neuhauser and Pacala (1999) so we will stick to it.

As in Ezoe's (1988) paper, we suppose that each of the two types produce seeds of a single weight $w$, and that the birth rate $\beta(w)$ and dispersal distribution are functions of $w$. In this context if we were to consider the competition of two types with fixed characteristics, it would be impossible to obtain quantitative results. Here we will circumvent that difficulty by instead considering the probability that a type $w$ can be invaded by $w+\varepsilon$ and let $\varepsilon \rightarrow 0$. This approach will allow us to use the machinery of Cox, Durrett, and Perkins (2011) to obtain explicit results, which we will illustrate with an example in which dispersal follows a two-dimensional geometric distribution.

A two dimensional lattice is most natural for studying the evolution of dispersal, but since the problem is mathematically simpler in dimensions $d \geq 3$, we will consider dimensions $d \geq 2$ here. To define the model we introduce two birth rates $\beta_{0}, \beta_{\varepsilon}>0$ and two dispersal distributions $p_{0}, p_{\varepsilon}$ in $\mathbb{Z}^{d}$ which we suppose satisfy for $0 \leq \varepsilon<\varepsilon_{0}$

$$
p_{\varepsilon} \text { is symmetric, irreducible, and has } p_{\varepsilon}(x) \leq C e^{-\kappa|x|} \text {. }
$$

where $\kappa>0$ is a constant. We think of $p_{0}$ and $\beta_{0}$ as being fixed, and $p_{\varepsilon}$ and $\beta_{\varepsilon}$ as perturbations, with $\beta_{\varepsilon} \rightarrow \beta_{0}$ and $p_{\varepsilon}(x) \rightarrow p_{0}(x)$ as $\varepsilon \rightarrow 0$ in a sense we will make precise later.

To formulate the transition rates of the process $\xi_{t}^{\varepsilon}$, we let

$$
f_{i}^{\varepsilon}(x)=\sum_{y \in \mathbb{Z}^{d}} p_{\varepsilon}(y-x) 1_{\{\xi(y)=i\}}
$$

be the fraction of neighbors of $x$ that are in state $i$, when sites are weighted according to the dispersal distribution $p_{\varepsilon}$. At $x \in \mathbb{Z}^{d}$

$$
\text { - } 0 \rightarrow 1 \text { at rate } \beta_{\varepsilon} f_{1}^{\varepsilon}(x)
$$

- $1 \rightarrow 0$ at rate $\beta_{0} f_{0}^{0}(x)$

To explain the first rate: a 1 at $y$ gives birth at rate $\beta_{\varepsilon}$ and sends its offspring to replace the 0 at $x$ with probability $p_{\varepsilon}(x-y)=p_{\varepsilon}(y-x)$. The explanation of the second rate is similar. When $\varepsilon$ is small, this is a perturbation of the voter model in which $1 \rightarrow 0$ at rate $\beta_{0} f_{1}^{0}(x)$ and $0 \rightarrow 1$ at rate $\beta_{0} f_{0}^{0}(x)$.

The voter model is easy to study because it is dual to a collection of coalescing random walks, a system in which particles move according to random walks that jump at rate $\beta_{0}$ with steps distributed according to $p_{0}$ and move independently until they hit, at which time they coalesce into one particle. If we let $\zeta_{t}$ be the set of sites 
occupied by 1's in the voter model, and $\hat{\zeta}_{t}$ be the coalescing random walk then the formal definition of the dual is

$$
P\left(\zeta_{t}^{A} \cap B \neq \emptyset\right)=P\left(A \cap \hat{\zeta}_{t}^{B} \neq \emptyset\right)
$$

where the superscripts indicate the starting configurations, i.e., $\zeta_{0}^{A}=A$ and $\hat{\zeta}_{0}^{B}=B$. Intuitively $\hat{\zeta}_{t}$ describes the genealogy of the opinions in the voter model. To be formal, for each $t$ we can define a dual $\hat{\zeta}_{s}^{(x, t)}$, which gives the site which $x$ traces its opinion back to at time $t-s$, and the overall dual comes from combining these: $\hat{\zeta}_{s}^{B}, 0 \leq s \leq t$ has the same distribution as $\cup_{x \in B} \hat{\zeta}_{s}^{(x, t)}, 0 \leq s \leq t$. The coalescence in the dual comes from the fact that if two sites trace their lineage back to the same site at the same time then the rest of their genealogies will be the same.

Duality is the key to the study of properties of the voter model. For example, under our assumption of symmetry and exponential tails, two random walks will eventually hit in two dimensions, see e.g., Spitzer (1976), so (2) implies that the voter model converges to complete consensus, i.e., for any finite set $B$ the probability that there are two different opinions in $B$ is $\leq P\left(\left|\hat{\zeta}_{t}^{B}\right| \geq 2\right) \rightarrow 0$, where we have written $|A|$ for the number of points in $A$. In $d \geq 3,(2)$ allows us to conclude that if we start with product measure with density $p$, i.e., sites are independently 1 or 0 with probabilities $p$ and $1-p$, then as $t \rightarrow \infty$ there is convergence to a nontrivial translation invariant stationary distribution $\nu_{p}$ in which the fraction of sites in state 1 is equal to $p$. For a more detailed explanation of the results in the last two paragraphs see the original paper by Holley and Liggett (1975).

To put our model in the framework of voter model perturbations of Cox, Durrett, and Perkins (2011), we will make the following assumption: there is a function $\Lambda$ such that

$$
\beta_{\varepsilon} p_{\varepsilon}(x)-\beta_{0} p_{0}(x)=\varepsilon^{2} \Lambda(x)+o\left(\varepsilon^{2}\right) .
$$

When we need to be more precise we will define

$$
\Lambda^{\varepsilon}(x)=\varepsilon^{-2}\left[\beta_{\varepsilon} p_{\varepsilon}(x)-p_{0}(x)\right]
$$

Here $\beta_{\varepsilon} p_{\varepsilon}(x)$ is the total rate at which an individual at site $y$ sends offspring to $y+x$, so $\Lambda_{\varepsilon}(x)$ can be thought of as the rate of change of this total rate as $\varepsilon \rightarrow 0$.

In terms of the original motivation we are comparing seeds with weight $w$ to those with weight $w+\varepsilon^{2}$. This parameterization in terms of $\varepsilon^{2}$ may seem unusual, but as we will now see it is convenient. If we speed up time at rate $\varepsilon^{-2}$ and scale space by $\varepsilon$, so that we are considering the process on a fine lattice $\varepsilon \mathbb{Z}^{d}$, then the coalescing random walks $\hat{\zeta}_{t}^{x}$ of the voter model converge to Brownian motions, and in addition changes in the state at each site due to the perturbation occur at a rate that is $O(1)$.

To motivate the next step, we recall the work of Durrett and Neuhauser (1994), who considered particle systems that were modified so that stirring (changing the values at adjacent sites) occurred at rate $\varepsilon^{-2}$ and the lattice was scaled to $\varepsilon \mathbb{Z}^{d}$. In the fast stirring situation and in the voter model perturbations, we can define a 
dual process where particles move according to Brownian motions, and at rate $O(1)$ branchings occur to include the new sites that we need know the states of in order to figure out the state of the process at $x$ at time $t$. The dual is somewhat complicated, so we refer to Cox, Durrett, and Perkins (2011) for a detailed description. However, the existence of this complicated object is the key to the proof of Theorem 1.

In the case of fast stirring things are simple because lineages do not coalesce. In our situation, particles in the dual branch into two at a constant rate to take into account situations for example where only a 1 at $y$ would change a 0 at $x$ to 1. If the two lineages generated later coalesce then $x$ and $y$ had the same state and nothing happened. In $d \geq 3$, there is positive probability that two random walks never coalesce, so we can use the same scaling of time $\left(\varepsilon^{-2}\right)$ and space $(\varepsilon)$. In $d=2$ the probability two random walks do not coalesce by time $t$ is $O(1 / \log t)$, so we need to further speed up time to $\tau_{\varepsilon}=\varepsilon^{-2} \log (1 / \varepsilon)$ so that the perturbation will have an effect. Having done this, we need to scale the lattice by $1 / \sqrt{\tau_{\varepsilon}}$ to have the genealogies converge to Brownian motion. Let

$$
\kappa_{\varepsilon}= \begin{cases}\varepsilon & d \geq 3 \\ \varepsilon /(\log (1 / \varepsilon))^{1 / 2} & d=2\end{cases}
$$

We will scale space to $\kappa_{\varepsilon} \mathbb{Z}^{d}$ and assume the following technical conditions hold for some $\varepsilon_{0}$ :

$$
\begin{aligned}
& \lim _{\varepsilon \rightarrow 0} \varepsilon^{2} \kappa_{\varepsilon}^{-2} \sum_{y \in \mathbb{Z}^{d}}\left|\Lambda^{\varepsilon}(y)-\Lambda(y)\right|=0 \\
& \sum_{y \in \mathbb{Z}^{d}} \sup _{\varepsilon \in\left(0, \varepsilon_{0} / 2\right]}\left|\Lambda^{\varepsilon}(y)\right|<\infty \\
& \Lambda(x)+\varepsilon_{0}^{-2} p_{0}(x)>0 \text { for all } x \text { such that } \Lambda(x) \neq 0
\end{aligned}
$$

The first two conditions are necessary to provide control over the convergence of the infinitely many sequences $p_{\varepsilon}(x) \rightarrow p_{0}(x)$. The last is needed to be able to rewrite the perturbation $\Lambda^{\varepsilon}(x)$ as a nonnegative function that can be interpreted as the flip rates of a Markov chain.

With these assumptions, methods from Cox, Durrett, and Perkins (2011) can be extended to give the following result. That paper is more than 100 pages long, and the details are even more complicated in $d=2$ so we content ourselves here to state the results and apply them to a concrete example. The mathematical details are still being worked out by Durrett and Remenik (2011).

For this and our next theorem we assume (1), (5), (6) and (7).

Theorem 1. As $\varepsilon \rightarrow 0$ the particle system on the fine lattice $\varepsilon \mathbb{Z}^{d}$ becomes deterministic and the density of occupied sites converges to the solution of

$$
\frac{\partial u}{\partial t}=\beta_{0} \frac{\sigma_{0}^{2}}{2} \Delta u+\beta_{0} m^{*} u(1-u), \quad u(0, x)=v(x)
$$


where $\sigma_{0}^{2}$ is the variance of the distribution $p_{0}$, and

$$
m^{*}= \begin{cases}\pi \sigma_{0}^{2} \sum_{y} \Lambda(y) a(y) & \text { if } d=2 \\ \sum_{y} \mathbb{P}\left(\tau_{y}=\infty\right) \Lambda(y) & \text { if } d \geq 3\end{cases}
$$

where $\tau_{y}=\inf \left\{t \geq 0: \hat{\zeta}^{y}(t)=\hat{\zeta}_{t}^{0}\right\}$ and $a(x)$ is the recurrent potential kernel for the random walk:

$$
a(x)=\int_{0}^{\infty} P\left(\hat{\zeta}_{t}^{0}=0\right)-P\left(\hat{\zeta}_{t}^{x}=0\right) d t .
$$

The interpretation of $m^{*}$ is that when $\varepsilon$ is small and the species with characteristics $\beta^{\varepsilon}$ and $p_{\varepsilon}$ is rare its local density increases at rate $m^{*} \varepsilon^{2}$.

Theorem 1 is one of many results in the probability literature that are "hydrodynamic limits," see e.g., Kipnis and Landim (1999). The intuition behind this result is that since the voter dynamics occur at a fast rate then in $d \geq 3$ when the density near $x$ at time $t$ is $u(t, x)$, the configuration near $x$ looks like the voter stationary distribution $\nu_{u}(t, x)$, and the reaction term is obtained by taking the expected value of the rate of change under this equilibrium. This is the same intuition as in the result of Durrett and Neuhauser (1994) but there product measure is the equilibrium for fast stirring so the reaction term reduces to the mean-field differential equation. In $d=2$ there is no stationary distribution, but we have an estimate on the probability that the duals from $x$ and $y$ chosen with probability $p_{0}(y-x)$ have not coalesced, and when they have not, the values at $x$ and $y$ at time $t$ are equal to 1 and 0 with probability $\approx u(t, x)(1-u(t, y))$ which is $\approx u(t, x)(1-u(t, x))$, since $y$ is close to $x$ on $\kappa_{\varepsilon} \mathbb{Z}^{2}$.

The next thing to explain is what we mean by "the particle system on the fine lattice $\varepsilon \mathbb{Z}^{d}$ becomes deterministic and the density of occupied sites converges to the solution of the PDE." To define the density of the particle system, we divide space in cubes of size $\varepsilon^{a}$ where $a$ is small and demand that for any $K<\infty$, with probability tending to 1 , the fraction of occupied sites is close to $u(t, z)$ for each cube with lower left corner $z \in \varepsilon^{a} \mathbb{Z}^{d} \cap[-K, K]^{d}$. Of course to achieve convergence of the particle system to the solution of the PDE, we have to assume that the initial condition of the particle system converges to $v(x)$ in this sense.

The reason for interest in Theorem 1 is that it allows us to prove:

Theorem 2. There is a $\varepsilon_{1}>0$ such that for every $\varepsilon \in\left(0, \varepsilon_{1}\right)$ the process $\xi_{t}^{\varepsilon}$ satisfies the following:

1. If $m^{*}>0$ then the 1's take over, i.e., starting from any initial configuration with infinitely many 1's, the system converges to all 1's.

2. If $m^{*}<0$ then the $0^{\prime}$ 's take over. 
When $m^{*}>0,(8)$ is called Fisher's (1937) equation for the advance of an advantageous gene, or the KPP equation, after Kolmogoroff, Petrovsky, and Piscounov (1937). A result of Aronson and Weinberger (1975) implies that there is a $c>0$ so that starting from any initial condition $v \not \equiv 0$

$$
\inf _{|x| \leq c t}|1-u(t, x)| \rightarrow 0
$$

As in Durrett and Neuhauser (1994) this provides input for a "block construction" to prove Theorem 2. Cox, Durrett, and Perkins (2011) generalizes the argument, which was initially given for a contact process with sexual reproduction, and fills some lacunae in the original proof.

\section{Application}

We will discuss now how Theorem 2 can be used to study the tradeoff between dispersal range and seed size in a concrete example. We begin by defining the dispersal distributions:

$$
\begin{aligned}
& p_{0}(x)=\left(K_{0}\right)^{d} \prod_{i=1}^{d}\left(\left[\frac{r_{0}}{r_{0}+1}\right]^{\left|x_{i}\right|}+\mathbf{1}_{\left\{x_{i}=0\right\}}\right) \\
& p_{\varepsilon}(x)=\left(K_{\varepsilon}\right)^{d} \prod_{i=1}^{d}\left(\left[\frac{r_{\varepsilon}}{r_{\varepsilon}+1}\right]^{\left|x_{i}\right|}+\mathbf{1}_{\left\{x_{i}=0\right\}}\right)
\end{aligned}
$$

where $K_{0}$ and $K_{\varepsilon}$ are normalizing constants given by

$$
K_{0}=\frac{1}{2\left(r_{0}+1\right)} \quad \text { and } \quad K_{\varepsilon}=\frac{1}{2\left(r_{\varepsilon}+1\right)} .
$$

This choice for the dispersal distribution corresponds to choosing each of the $d$ coordinates according to a (modified) two-sided geometric distribution, where we have doubled the mass at the origin to simplify calculations. We observe that the mean dispersal ranges of $p_{0}$ and $p_{\varepsilon}$ (computed with respect to the norm $|x|=\left|x_{1}\right|+\ldots+\left|x_{d}\right|$ ) are $d r_{0}$ and $d r_{1}^{\varepsilon}$, respectively. We also have

$$
\sigma_{0}^{2}=r_{0}\left(2 r_{0}+1\right)
$$

Since the parameter $r$ is a decreasing function of seed size $w$, we can use $r$ as our variable instead of $w$. To each $r>0$ we associate a birth rate $\beta(r)$, so that individuals with dispersal range $r$ will give birth at rate $\beta(r)$. We will assume that $\beta(r)$ is decreasing and has two continuous derivatives.

To have an explicit example we will suppose that

$$
r_{\varepsilon}=r_{0}\left(1+\varepsilon^{\lambda}\right) \text { for some } \lambda>2 .
$$


Using Taylor's Theorem on the function $x \mapsto \beta\left(r_{0}(1+x)\right)$ and then setting $x=\varepsilon^{\lambda}$ we deduce that

$$
\beta_{1}^{\varepsilon}=\beta_{0}\left(1-c\left(r_{0}\right) \varepsilon^{\lambda}\right)+O\left(\varepsilon^{2 \lambda}\right) \quad \text { with } c\left(r_{0}\right)=-r_{0} \frac{\beta^{\prime}\left(r_{0}\right)}{\beta\left(r_{0}\right)}>0 .
$$

With this parametrization we can determine the behavior of $\xi_{t}^{\varepsilon}$ for given dispersal distributions and given choices of $r_{0}, \beta_{0}, c$, and $\varepsilon$.

The problem here is to look for evolutionary stable strategies (ESS), which correspond to choices of $r$ for which a resident population with this dispersal distance cannot be invaded by a mutant with a slightly different one. In our case, if we compute the relative growth rate $m^{*}$ as a function of $r_{0}$ then the only ESS are those where $m^{*}\left(r_{0}\right)=0$ and $m^{*}$ is decreasing at $r_{0}$. To see this, suppose that we have a resident population characterized by some type $r=\bar{r}$ and we introduce small mutations at a slow rate. If the mutant has a larger $r$ then with our parametrization the resident has type $r_{0}=\bar{r}$ and the mutant has type $r_{\varepsilon} \geq r_{0}$, and thus if $m^{*}$ is positive at $\bar{r}$ the mutant will take over. Conversely, if the mutant has a smaller $r$ then we need to set $r_{0}$ to be the mutant type and $r_{\varepsilon}=\bar{r}$, and thus if $m^{*}$ is negative to the left of $\bar{r}$, the mutant will take over.

In order to compute $\Lambda(x)$, write $f_{x}\left(\varepsilon^{\lambda}\right)=\beta_{0}\left(1-c\left(r_{0}\right) \varepsilon^{\lambda}\right) p_{1}^{\varepsilon}(x)$ for $x \in \mathbb{Z}^{d}$ and observe that $\varepsilon^{2} \Lambda^{\varepsilon}(x)=\beta_{1}^{\varepsilon} p_{1}^{\varepsilon}(x)-\beta_{0} p_{0}(x)=f_{x}\left(\varepsilon^{\lambda}\right)-f_{x}(0)$. After some tedious calculations one gets

$$
f_{x}^{\prime}(0)=\left[-c\left(r_{0}\right)-d \frac{r_{0}}{r_{0}+1}+\frac{|x|}{r_{0}+1}\right] \beta_{0} p_{0}(x)
$$

so letting $\alpha\left(r_{0}\right)=\left(r_{0}+1\right) c\left(r_{0}\right)+d r_{0}$ we get that

$$
f_{x}\left(\varepsilon^{\lambda}\right)-f_{x}(0)=\varepsilon^{\lambda} \frac{\beta_{0}}{r_{0}+1} p_{0}(x)\left[|x|-\alpha\left(r_{0}\right)\right]+\frac{\varepsilon^{2 \lambda}}{2} f_{x}^{\prime \prime}(h)
$$

for some $h$ between 0 and $\varepsilon^{\lambda}$. Therefore

$$
\Lambda(x)=\frac{\beta_{0}}{r_{0}+1} p_{0}(x)\left[|x|-\alpha\left(r_{0}\right)\right] \quad \text { and } \quad \Lambda_{\varepsilon}(x)=\Lambda(x)+O\left(\varepsilon^{2(\lambda-1)}\right) .
$$

Moreover, the $O\left(\varepsilon^{2(\lambda-1)}\right)$ term above equals $\varepsilon^{2(\lambda-1)} f_{x}^{\prime \prime}(h) / 2$ and it is clear that $\sum_{x} f_{x}^{\prime \prime}(h)$ is bounded uniformly away from infinity for small $h$, whence we deduce that (5) and (6) hold. Checking that (7) holds is straightforward.

Now we need to compute $m^{*}$, and we will do this only in the biologically interesting case $d=2$. By (3) in page 122 of Spitzer (1976) we have

$$
a(x)=\frac{1}{(2 \pi)^{2}} \int_{[-\pi, \pi]^{2}} \frac{1-e^{-i x \cdot \theta}}{1-\phi(\theta)} d \theta=\frac{1}{(2 \pi)^{2}} \int_{[-\pi, \pi]^{2}} \frac{1-\cos (x \cdot \theta)}{1-\phi(\theta)} d \theta,
$$


where $\phi$ is the characteristic function of $p_{0}$ and we used the fact that $a(x)$ is real. More tedious but simple calculations give

$$
\begin{aligned}
\phi(\theta) & =\frac{1}{4}\left[\frac{1}{r_{0}+1-r_{0} e^{i \theta_{1}}}+\frac{1}{r_{0}+1-r_{0} e^{-i \theta_{1}}}\right]\left[\frac{1}{r_{0}+1-r_{0} e^{i \theta_{2}}}+\frac{1}{r_{0}+1-r_{0} e^{-i \theta_{2}}}\right] \\
& =\left[\frac{r_{0}+1-r_{0} \cos \left(\theta_{1}\right)}{\left(r_{0}+1\right)^{2}-2 r_{0}\left(r_{0}+1\right) \cos \left(\theta_{1}\right)+r_{0}^{2}}\right]\left[\frac{r_{0}+1-r_{0} \cos \left(\theta_{2}\right)}{\left(r_{0}+1\right)^{2}-2 r_{0}\left(r_{0}+1\right) \cos \left(\theta_{2}\right)+r_{0}^{2}}\right] .
\end{aligned}
$$

This can be used in the above formula to obtain $a(x)$ by numerical integration, and then use the formula for $\Lambda$ to compute $m^{*}$, see Figure 1.

\section{References}

Aronson, D.G. and Weinberger, H.F. (1975) Nonlinear diffusion in population genetics, combustion, and nerve pulse propagation. Pages 5-49 in Partial Differential Equations and Related Topics. Springer Lecture Notes in Math 446, Springer, New York.

Cohen, D., and Levin, S.A. (1991) Dispersal in patchy environments: The effects of temporal and spatial structure. Theor. Pop. Biol. 39, 63-99

Cox, J.T., Durrett, R., and Perkins, E.A. (2011) Voter model perturbations and reaction diffusion equations. arXiv:1103.1676

Durrett, R., and Remenik, D. (2011) Voter model perturbations in two dimensions. Manuscript in preparation.

Durrett, R. and Neuhauser, C. (1994) Particle systems and reaction-diffusion equations. Ann. Probab. 22, 289-333

Ezoe, H. (1998) Optimal dispersal range and seed size in a stable environment. J. Theor. Biol. 190, 287-293

Fisher, R. A. (1937) The wave of advance of advantageous genes. Ann. Eugen. 7, 355-369

Hamilton, W.D., and May, R.M. (1977) Dispersal in stable habitats. Nature. 269, 578-581

Hiebeler, D. (2004) Competition between near and far dispersers in spatially structured habitats. Theor. Pop. Biol. 66, 205-218

Holley, R.A., and Liggett, T.M. (1975) Ergodic theorems for weakly interacting infinite systems and the voter model. Ann. Probab. 3 (1975), 643-663

Kipnis, C., and Landim, C. (1999) Scaling limits of particle systems. Springer, New York 
Kolmogoroff, A., Petrovsky, P., and Piscounov, N. (1937) Étude de l'equations de la diffusion avec croissance de la quantité de matière et son application a un problème biologique. Bull. Univ. Etat Moscou, Ser. Int. Set: A. 1, 1-25

Levin, S.A., Cohen, D., and Hastings, A. (1984) Dispersal strategies in patchy environments. Theor. Pop. Biol. 26, 165-191

Levin, S.A. and Muller-Landau, H.C. (2000) The evolution of dispersal and seed size in plant communities. Evol. Ecol. Res. 2, 409-435

Levin, S.A., Muller-Landau, H.C., Nathan, R., and Chave, J. (2003) The ecology and evolution of seed dispersal: A theoretical perscpective. Annu. Rev. Ecol. Syst. 34, $575-604$

Ludwig, D., and Levin, S.A. (1991) Evolutionary stability of plant communities and maintenance of multiple dispersal types. Theor. Pop. Biol. 40, 285-307

Neuhauser, C., and Pacala, S.W. (1999) An explicitly spatial version of the LotkaVolterra model with interspecific competition. Ann. Appl. Probab. 9, 1226-1259.

Spitzer, F. (1976) Principles of random walks, Second edition. Graduate Texts in Mathematics, Vol. 34, Springer-Verlag, New York 


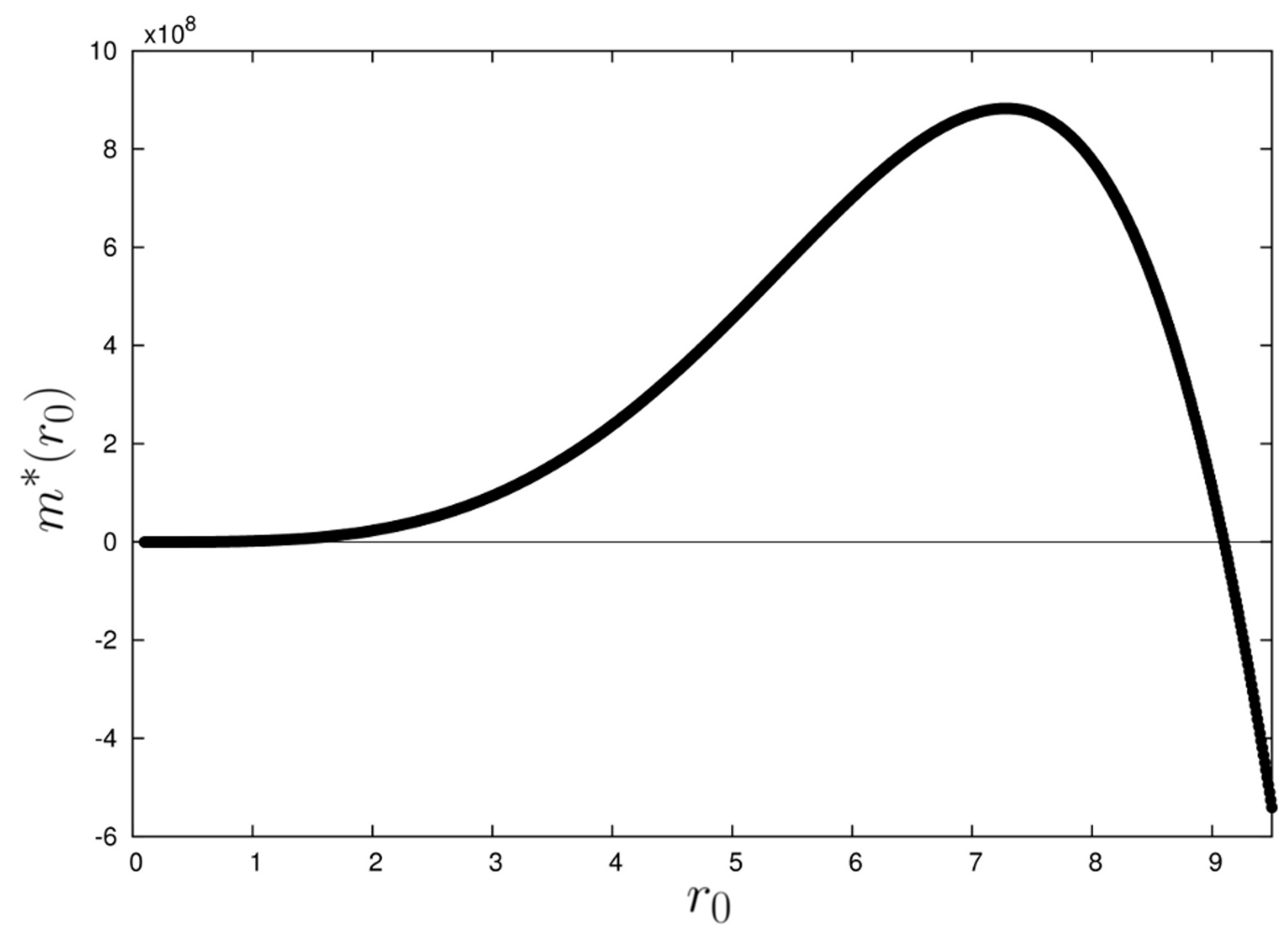

Figure 1: Numerical computation of the constant $m^{*}\left(r_{0}\right)$ in Theorem 1.2 for Example 1.5 with $\beta_{0}=1$ and $c\left(r_{0}\right)=1.8+0.2 r_{0}$. The function has two zeros, the first one (barely visible on the graph at $r_{0}=0.468$ and the second one at $r_{0}=9.1$. The function is increasing at the first zero and decreasing at the second which is the ESS. 